\title{
Energy-Momentum Density's Conservation Law of Electromagnetic Field in Rindler Space-time
}

\author{
Sangwha-Yi* \\ Department of Math, Taejon University 300-716
}

${ }^{*}$ Corresponding Author: Sangwha-Yi, Department of Math, Taejon University 300-716

\begin{abstract}
We find the energy-momentum density of electromagnetic field by energy-momentum tensor of electromagnetic field in Rindler space-time. We find the energy-momentum density's conservation law of electromagnetic field in Rindler spacetime.
\end{abstract}

Keywords: The general relativity theory, The Rindler spacetime, Energy-momentum density, Conservation law

PACS: 04,04.90. $+e, 41.20$

\section{INTRODUCTION}

Our article's aim is that we find the energy-momentum density of electromagnetic field by energymomentum tensor of electromagnetic field in Rindler space-time. We find the energy-momentum density's conservation law of electromagnetic field in Rindler space-time.

In inertial frame, the energy-momentum tensor $T^{\mu \nu}$ of the electromagnetic field is

$T^{\mu \nu}=\frac{1}{4 \pi c}\left(F^{\mu}{ }_{\rho} F^{v \rho}-\frac{1}{4} \eta^{\mu \nu} F_{\rho \sigma} F^{\rho \sigma}\right)$

In this time, in inertial frame, Faraday tensors $F^{\mu \nu}, F_{\mu v}$ are

$F^{\mu \nu}=\left(\begin{array}{cccc}0 & E_{x} & E_{y} & E_{z} \\ -E_{x} & 0 & B_{z} & -B_{y} \\ -E_{y} & -B_{z} & 0 & B_{x} \\ -E_{z} & B_{y} & -B_{x} & 0\end{array}\right), F_{\mu \nu}=\left(\begin{array}{cccc}0 & -E_{x} & -E_{y} & -E_{z} \\ E_{x} & 0 & B_{z} & -B_{y} \\ E_{y} & -B_{z} & 0 & B_{x} \\ E_{z} & B_{y} & -B_{x} & 0\end{array}\right)$

Hence, the energy density $p_{f}{ }^{0}$ and the momentum density $\vec{p}_{f}$ of electromagnetic field are

$T^{00}=p_{f}^{0}=\frac{E^{2}+B^{2}}{8 \pi c}, T^{0 i}=\vec{p}_{f}=\frac{\vec{E} \times \vec{B}}{4 \pi c}, i=1,2,3$

$|\vec{E}|=E,|\vec{B}|=B$

In inertial frame, the energy-momentum conservation law of electromagnetic field is by Noether theorem,

$$
\begin{aligned}
& T^{\mu v}{ }_{v}=T^{00}{ }_{0}+T^{0 i},_{i}, i=1,2,3 \\
& =\frac{1}{c} \frac{\partial}{\partial t}\left(\frac{E^{2}+B^{2}}{8 \pi c}\right)+\vec{\nabla} \cdot\left(\frac{\vec{E} \times \vec{B}}{4 \pi c}\right)=0
\end{aligned}
$$

\section{EnERgy-Momentum Density's CONSERVATION EleCtromagnetic Field IN RindLer SPACETIME}

Rindler space-time is

$$
d \tau^{2}=\left(1+\frac{a_{0} \xi^{1}}{c^{2}}\right)\left(d \xi^{0}\right)^{2}-\frac{1}{c^{2}}\left[\left(d \xi^{1}\right)^{2}+\left(d \xi^{2}\right)^{2}+\left(d \xi^{3}\right)^{2}\right]=-\frac{1}{c^{2}} g_{\mu \nu} d \xi^{\mu} d \xi^{v}
$$

In Rindler space-time, the energy-momentum tensor $T^{\mu v}$ of the electromagnetic field is 
$T^{\mu \nu}=\frac{1}{4 \pi C}\left(F^{\mu}{ }_{\rho} F^{v \rho}-\frac{1}{4} g^{\mu \nu} F_{\rho \sigma} F^{\rho \sigma}\right)$

In this time, in Rindler space-time, Faraday tensor $F_{\xi}{ }^{\mu v}$ is[2]

$$
F_{\xi}^{\mu \nu}=\left(\begin{array}{cccc}
0 & E_{\xi^{1}} & E_{\xi^{2}} & E_{\xi^{3}} \\
-E_{\xi^{1}} & 0 & \left(1+\frac{a_{0} \xi^{1}}{c^{2}}\right) B_{\xi^{3}} & -\left(1+\frac{a_{0} \xi^{1}}{c^{2}}\right) B_{\xi^{2}} \\
-E_{\xi^{2}} & -\left(1+\frac{a_{0} \xi^{1}}{c^{2}}\right) B_{\xi^{3}} & 0 & \left(1+\frac{a_{0} \xi^{1}}{c^{2}}\right) B_{\xi^{1}} \\
-E_{\xi^{3}} & \left(1+\frac{a_{0} \xi^{1}}{c^{2}}\right) B_{\xi^{2}} & -\left(1+\frac{a_{0} \xi^{1}}{c^{2}}\right) B_{\xi^{1}} & 0
\end{array}\right)
$$

In Rindler space-time, Faraday tensor $F_{\xi \mu \nu}$ is[2]

$$
F_{\xi_{\mu \nu}}=\left(\begin{array}{cccc}
0 & -\left(1+\frac{a_{0} \xi^{1}}{c^{2}}\right) E_{\xi^{1}} & -\left(1+\frac{a_{0} \xi^{1}}{c^{2}}\right) E_{\xi^{2}} & -\left(1+\frac{a_{0} \xi^{1}}{c^{2}}\right) E_{\xi^{3}} \\
\left(1+\frac{a_{0} \xi^{1}}{c^{2}}\right) E_{\xi^{1}} & 0 & B_{\xi^{3}} & -B_{\xi^{2}} \\
\left(1+\frac{a_{0} \xi^{1}}{c^{2}}\right) E_{\xi^{2}} & -B_{\xi^{3}} & 0 & B_{\xi^{1}} \\
\left(1+\frac{a_{0} \xi^{1}}{c^{2}}\right) E_{\xi^{3}} & B_{\xi^{2}} & -B_{\xi^{1}} & 0
\end{array}\right)
$$

Hence, the energy density $p_{\xi_{f}}{ }^{0}$ and the momentum density $\vec{p}_{\xi_{f}}$ of electromagnetic field are in Rindler space-time.

$$
\begin{aligned}
& T^{00}=p_{\xi_{f}}{ }^{0}=\frac{1}{\left(1+\frac{a_{0} \xi^{1}}{c^{2}}\right)} \frac{E_{\xi}{ }^{2}+B_{\xi}{ }^{2}}{8 \pi c} \\
& T^{0 i}=\vec{p}_{\xi_{f}}=\frac{\vec{E}_{\xi} \times \vec{B}_{\xi}}{4 \pi c}, i=12,3 \\
& \left|\vec{E}_{\xi}\right|=E_{\xi},\left|\vec{B}_{\xi}\right|=B_{\xi}
\end{aligned}
$$

In Rindler space-time, the energy-momentum conservation law of electromagnetic field is by Noether theorem,

$$
\begin{aligned}
& T_{i v}^{\mu v}=T_{; 0}^{00}+T_{; i}^{0 i}=T_{i v}^{0 v}, \quad i=1,2,3 \\
& =\frac{\partial T^{0 v}}{\partial x^{v}}+\Gamma_{\sigma v}^{0} T^{\sigma v}+\Gamma_{\sigma v}^{v} T^{0 \sigma}
\end{aligned}
$$

In this time, affine connections are in Rindler space-time

$$
\Gamma_{00}^{1}=-\left(1+\frac{a_{0}}{c^{2}} \xi^{1}\right) \frac{a_{0}}{c^{2}}, \Gamma_{10}^{0}=\Gamma_{01}^{0}=\frac{1}{\left(1+\frac{a_{0} \xi^{1}}{c^{2}}\right)} \frac{a_{0}}{c^{2}}
$$

Hence, in Rindler space-time, the energy-momentum conservation law of electromagnetic field is

$$
\begin{aligned}
& T_{i v}^{\mu v}=T_{; 0}^{00}+T_{; i}^{0 i}=T_{i v}^{0 v} \\
& =\frac{\partial T^{0 v}}{\partial x^{v}}+\Gamma_{\sigma v}^{0} T^{\sigma v}+\Gamma^{v}{ }_{\sigma v} T^{0 \sigma} \\
& =\frac{\partial T^{0 v}}{\partial x^{v}}+3 \Gamma_{01}^{0} T^{01}
\end{aligned}
$$




$$
\begin{aligned}
& =\frac{1}{\left(1+\frac{a_{0} \xi^{1}}{c^{2}}\right)} \frac{1}{c} \frac{\partial}{\partial \xi^{0}}\left(\frac{E_{\xi}^{2}+B_{\xi}^{2}}{8 \pi c}\right)+\vec{\nabla}_{\xi} \cdot\left(\frac{\vec{E}_{\xi} \times \vec{B}_{\xi}}{4 \pi c}\right)+3 \frac{1}{\left(1+\frac{a_{0} \xi^{1}}{c^{2}}\right)} \frac{a_{0}}{c^{2}} \frac{1}{4 \pi c}\left(E_{\xi^{3}} B_{\xi^{2}}-E_{\xi^{2}} B_{\xi^{3}}\right)=0 \\
& \vec{\nabla}_{\xi}=\left(\frac{\partial}{\partial \xi^{1}}, \frac{\partial}{\partial \xi^{2}}, \frac{\partial}{\partial \xi^{3}}\right) \\
& \left|\vec{E}_{\xi}\right|=E_{\xi},\left|\vec{B}_{\xi}\right|=B_{\xi}
\end{aligned}
$$

\section{CONCLUSION}

We find the energy-momentum density's conservation law of electromagnetic field in Rindler spacetime.

\section{REFERENCES}

[1] S.Yi, "Electromagnetic field equation and Lorentz Gauge in Rindler Space-time",The African Review of Physics, 11,33(2016)-INSPIRE-HEP

[2] S.Yi, "Einstein's Notational Equation of Electro-Magnetic Field Equation in Rindler space-time", International Journal of Advanced Research in Physical Science,6,5(2019)pp 4-6

[3] S.Weinberg,Gravitation and Cosmology(John wiley \& Sons,Inc,1972)

[4] W.Rindler, Am.J.Phys.34.1174(1966)

[5] P.Bergman,Introduction to the Theory of Relativity(Dover Pub. Co.,Inc., New York,1976), Chapter V

[6] C.Misner, K,Thorne and J. Wheeler, Gravitation(W.H.Freedman \& Co.,1973)

[7] S.Hawking and G. Ellis, The Large Scale Structure of Space-Time(Cam-bridge University Press,1973)

[8] R.Adler,M.Bazin and M.Schiffer,Introduction to General Relativity(McGraw-Hill,Inc.,1965)

[9] A.Miller, Albert Einstein's Special Theory of Relativity(Addison-Wesley Publishing Co., Inc., 1981)

[10] W.Rindler, Special Relativity(2nd ed., Oliver and Boyd, Edinburg,1966)

[11] J.W.Maluf and F.F.Faria,"The electromagnetic field in accelerated frames":Arxiv:gr-qc/1110.5367v1(2011)

[12] Massimo Pauri, Michele Vallisner, "Marzke-Wheeler coordinates for accelerated observers in special relativity":Arxiv:gr-qc/0006095(2000)

Citation: Sangwha-Yi, (2019). Energy-Momentum Density's Conservation Law of Electromagnetic Field in Rindler Space-time. International Journal of Advanced Research in Physical Science (IJARPS) 6(7), pp.2729, 2019.

Copyright: (c) 2019 Authors, this is an open-access article distributed under the terms of the Creative Commons Attribution License, which permits unrestricted use, distribution, and reproduction in any medium, provided the original author and source are credited. 